\title{
Relire et réécrire l'Écosse : le mythe égyptien d'Hector Boèce à Charles Cordiner
}

Reinterpreting Scotland: The Egyptian Myth from Hector Boece to Charles Cordiner

\section{Sylvie Kleiman-Lafon}

\section{(2) OpenEdition \\ Journals}

Édition électronique

URL : http://journals.openedition.org/etudesecossaises/766

DOI : 10.4000/etudesecossaises.766

ISSN : 1969-6337

Éditeur

UGA Éditions/Université Grenoble Alpes

Édition imprimée

Date de publication : 15 avril 2013

Pagination : 29-45

ISBN : 978-2-84310-246-2

ISSN : $1240-1439$

\section{Référence électronique}

Sylvie Kleiman-Lafon, «Relire et réécrire l'Écosse : le mythe égyptien d'Hector Boèce à Charles

Cordiner », Études écossaises [En ligne], 16 | 2013, mis en ligne le 15 avril 2014, consulté le 16 mars 2021. URL : http://journals.openedition.org/etudesecossaises/766 ; DOI : https://doi.org/10.4000/ etudesecossaises.766 


\section{Relire et réécrire l'Écosse : le mythe égyptien d'Hector Boèce à Charles Cordiner}

Lorsque James MacPherson publie pour la première fois les plus célèbres faux de la littérature écossaise ${ }^{1}$, il inscrit sa démarche dans une tentative plus vaste d'appropriation ou de réappropriation du passé historique de l'Écosse. Cette entreprise n'est pas propre au contexte politique particulier de l'Acte d'union, ni même aux tentatives désespérées du prétendant; elle n'est d'ailleurs pas propre à l'Écosse elle-même. Au XVI ${ }^{\mathrm{e}}$ siècle, la France découvre son passé gaulois et trouve dans cette nouvelle mythologie des origines la preuve d'une ancienneté prestigieuse qui remonterait au déluge ${ }^{2}$. Au début du XVIII ${ }^{\mathrm{e}}$ siècle, Paul-Yves Pezron écrit encore :

On ne pourra donc pas me blâmer, si je dis que Gomer, qui selon l'Écriture a été le fils aîné de Faphet, et le petit-fils de Noé, doit être regardé comme le premier et le véritable père des peuples gaulois, auxquels les Grecs donnent communément le nom de Galates ${ }^{3}$.

Dans le cas de l'Écosse, la question de l'origine du peuple écossais se fait jour à la fin du Moyen Âge et à la Renaissance avant d'être reprise avec plus ou moins de conviction par les historiographes du XVIII ${ }^{\mathrm{c}}$ siècle qui cherchent, pour des raisons diverses, à affirmer la prestigieuse antiquité de l'Écosse en la reliant à la Grèce ancienne, à Rome ou à l'Égypte. Au-delà des mythes fondateurs proposés par les historiens de la Renaissance écossaise, les antiquaires et les linguistes des Lumières se fondent

1. J. MacPherson, Fragments of Ancient Poetry, Collected in the Highlands of Scotland, and translated from the Galic or Erse Language, Édimbourg, Hamilton \& Balfour, 1760.

2. Voir par exemple : J. Lemaire de Belges, Les Illustrations de Gaule et singularités de Troye, Paris, 1512, ou J. de Cassan, Les Dynasties, ou Traité des anciens rois des Gaulois et des François depuis le Déluge successivement jusqu'au roi Mérovée, Paris, s. d. Concernant la réécriture du passé de la Gaule, on lira également: A. Talon (éd.), Le Sentiment national dans l'Europe méridionale aux XVI et XVII siècles, Madrid, Casa Velasquez, 2007.

3. P.-Y. Pezron, Antiquité de la nation et de la langue des Celtes, autrement appelez Gaulois, Paris, Jean Boudot, 1703. L'ouvrage est traduit en anglais par D. Jones et publié en 1706 sous le titre: The Antiquities of Nations; more particularly of the Celte or Gauls, taken to be originally the same people as our ancient Britains, Londres, S. Ballard, 1706. 
sur les indices matériels dont ils disposent pour réécrire le passé d'une région qui souffre d'être aux confins de l'Europe et du Royaume-Uni.

La description et l'interprétation de certaines pierres levées ornées de bas-reliefs offre un exemple de cette réécriture complexe.

\section{Les premières versions et les reprises du mythe fondateur}

Dans le cas de l'Écosse, les premières théories fondatrices s'appuyant sur un substrat à la fois biblique et orientalisant remontent aux chroniqueurs médiévaux. William Matthews fait remonter l'apparition du mythe égyptien aux chroniques pictes rédigées avant l'an mille et sa première utilisation politique à la confiscation de la pierre de Scone par Édouard ${ }^{\mathrm{I}}{ }^{\mathrm{r}}$, qui la transporte à Westminster et en fait la pierre de couronnement des souverains d'Angleterre ${ }^{4}$. Par la suite, Sir Thomas Gray y consacre une partie de la chronique qu'il rédige de la prison d'Édimbourg en $1355^{5}$; John of Fordun fait de même dans sa Chronica Gentis Scotorum rédigée quelques années plus tard, probablement entre 1384 et 1387, et continuée $\mathrm{au} \mathrm{XV}^{\mathrm{e}}$ siècle par Walter Bower ${ }^{6}$. Au XVI ${ }^{\mathrm{e}}$ siècle, l'historien d'Aberdeen Hector Boèce reprend dans le détail ce récit fondateur dans Scotorum Historice a prima gentis origine ${ }^{7}$. Comme le souligne Colin Kidd, il s'agit alors de concurrencer le mythe troyen des origines du royaume britannique (échafaudé par le chroniqueur Geoffrey de Monmouth et adopté par les Plantagenêt) et de s'en démarquer :

The community of Scotland supported the construction of a Scottish national "counter-mythology" in response to Edward I's reliance on the history of Geoffrey of Monmouth as a basis for his claims when the Anglo-Scottish dispute was referred to the arbitration of Pope Boniface VIII in 1301. According to Edward I's interpretation of Galfridian history, Brutus had been the first king of the whole empire of Britain, and had bequeathed the Kingdom of England and sovereignty over the whole island to his eldest son Locrinus, and Scotland and Wales to his younger sons, Albanacht and Camber. The Scots

4. W. Matthews, «The Egyptians in Scotland: The Political History of a Myth», Viator, 1, 1970, p. 289-306.

5. Sir Th. Gray, Scalacronica (1355), Édimbourg, pour le Maitland Club, 1836.

6. J. of Fordun, Chronica Gentis Scotorum (Scotichronicon). La première édition imprimée parait dans le troisième volume des Scriptores Quindecim de Th. Gale (1691). L'ouvrage ressort en latin en 1759 — augmenté de la suite écrite par Walter Bower — sous l'impulsion l'historien écossais Walter Goodall (Édimbourg, Thomas Flaminius, 1759).

7. H. Boèce, Scotorum Historia a prima gentis origine, Paris, 1526; traduction anglaise en 2 vols de R. Hollinshed, The Description of Scotland Written by H. Beothius in Latin... Translated into the Scotish [sic] Speech by John Belledon... and now finally into English by R. H., Londres, Harrison 1577. 
replied with their own pseudo-history of national independence, making use of royal genealogies and origin myths ${ }^{8}$.

Le point qui retient l'attention de tous ces chroniqueurs et surtout de Boèce est celui du peuplement primitif de l'Écosse, dont ils ont à cœur d'affirmer l'ancienneté et font remonter à l'Antiquité mosaïque. Avant même d'en retracer l'histoire, Boèce s'attache à donner une preuve tangible de ce qu'il s'apprête à avancer et relie explicitement l'écriture des Pictes aux hiéroglyphes observés en Égypte :

They used at the first the rites and manners of the Ægyptians from whence they came, and in all their private affaires they used not to write with common letters, as other nations did; but rather with ciphers and figures of creatures made in the manner of letters, as their epitaphes upon their toomes and sepulchers remaining among us hitherto declare. Nevertheless in our times, this hieroglyphicall manner of writing (I know not by what means) is perished and lost ${ }^{9}$.

Boèce parle ici des pierres levées, alors encore visibles en grand nombre sur le territoire écossais; il ancre l'hypothèse égyptienne dans le paysage, annonçant les descriptions et les interprétations du XVIII ${ }^{\mathrm{e}}$ siècle. Il expose ensuite le récit de cette origine, dont le point de départ remonte au personnage de Gathelus, que certains chroniqueurs postérieurs présentent comme un citoyen grec fils de Cecrops, fondateur d'Athènes et que d'autres font fils d'Argus Neacus, quatrième souverain d'Argives. Banni par son père à la suite de troubles en Macédoine, Gathelus se rend en Égypte où le Pharaon l'engage comme soldat dans les conflits qui l'opposent aux Éthiopiens à Memphis. Gathelus combat victorieusement sous les ordres de Moïse et reçoit deux présents prestigieux des mains du Pharaon en récompense de sa bravoure : la ville de Thèbes et la main de la fille du souverain. C'est par elle que le chroniqueur écossais établit un lien historique et étymologique entre l'Écosse et l'Égypte :

Here you must understand, that Pharaohs daughter, which Gathelus thus married, was called Scota, on whome such as came the posteritie of that nation were afterwards, and are at this present day called Scoti, that is to say Scotishmen, and the land where they inhabit Scotia, that is to say Scotland ${ }^{\mathbf{1 0}}$.

Fuyant l'Égypte après que les dix plaies se furent abattues sur elle, Gathelus et les siens cheminent pendant plusieurs années le long des côtes africaines avant de longer la côte occidentale de l'Espagne et de fonder, au

8. C. Kidd, Subverting Scotland's Past, Cambridge, Cambridge University Press, 1993, p. 17.

9. H. Boèce, Description of Scotland, ouvr. cité, p. 21.

10. Ibid., p. 29. 
nord, la ville de Brigantia que Boèce identifie à Compostelle. Il y érige des murailles, construit habitations et édifices publics, édicte des lois : Gathelus est présenté comme un bâtisseur et un civilisateur, à l'instar des pharaons égyptiens. L'enracinement dans un territoire, lié explicitement sous la plume de Boèce à la possession du sol et à la fonction législative s'exprime plus clairement encore à travers un objet dont la dimension politique n'échappe ni au chroniqueur de la Renaissance ni aux historiens du XVIII ${ }^{\mathrm{e}}$ siècle : la pierre de Scone.

Gathelus having peace with his neighbours, sat upon his marble stone in Brigantia, where he gave laws, and ministered Justice unto his people, thereby to maintain them in wealth and quietness. This stone was in fashion like a seat or chaire, having such a fatale destinie, as the Scots say, following it that wheresoever it should be found, there should the Scotishmen reign and have the supreme governance. Whereof it came to passe, that first in Spaine, after in Ireland, and then in Scotland, the kings which ruled over the Scotishmen, received the crowne sitting on that stone, untill the time of Robert, the first King of Scotland ${ }^{11}$.

Cette pierre scelle l'ancrage territorial du peuple écossais, mais représente aussi la promesse de conquêtes à venir. Boèce précise d'ailleurs que Gathelus, enhardi par la prospérité de son peuple, envoie ses deux fils, Hiberus et Himecus, trouver de nouvelles terres en Irlande (nommée pour cette raison Hibernia). Quelques années plus tard, Hiberus, las des heurts qui opposent ses «Écossais» aux autochtones irlandais retourne en Espagne et à la mort de son père décide de conduire les volontaires jusqu'aux îles Hébrides et aux îles de la côte ouest des Highlands : "The part where they first began to settle themselves, they named Argathelia, after the name of their first captain and guide Gathelus, but the inhabitants at this day call it Arguile ${ }^{12}$.»

Par la suite, les chroniqueurs qui ont succédé à Fordun et Boèce n’ont repris l'histoire de la sortie d'Égypte que pour la ravaler au rang de fable réputée colportée par des moines ${ }^{13}$. C'est le cas de Thomas Urquhart et George Buchanan au XVII ${ }^{\mathrm{e}}$ siècle, puis au siècle suivant de William Nicolson, Robert Sibbald, William Duncan, David Scott, Alexander

11. Ibid., p. 30 .

12. Ibid.

13. Voir J. Wallace, An Account of the Islands of Orkney, to which is added an Essay on the Thule of the Ancients, Londres, J. Tonson, 1700, p. 181 : «We need not have recourse, for the rise of the name Scot, to the fabulous account of the monks, who needs have it from one Scota, Pharaoh's daughter married to Gathelus.» 
Gordon ou William Maitland ${ }^{\mathbf{1 4}}$. Au XVIII ${ }^{\mathrm{e}}$ siècle, seuls quelques auteurs reprennent la thèse de l'origine égyptienne du peuple écossais, comme le jacobite James Wallace, James Brome, Thomas Innes ou Charles Cordiner ${ }^{15}$. Pour Colin Kidd, le «mythistoire» égyptien ne sera que très marginalement repris par les historiens whigs ou jacobites au moment de l'Union et pendant les décennies qui suivirent. L'attention se déplaça sur la présence romaine, notamment le mur d'Hadrien et d'autres vestiges comme le temple d'Arthur's Oven. Les preuves archéologiques de la résistance aux conquêtes successives devinrent alors plus importantes que celle de l'ancienneté de la nation écossaise, et même si certains historiens entendirent démontrer que l'Acte d'union avait été signé par deux couronnes d'égale stature et ne constituait pas une réintégration de l'Écosse dans le giron britannique, le mythe égyptien des origines fut oublié au profit d'autres éléments, plus récents, associés plus directement (et plus sérieusement) à la longévité de l'indépendance de la couronne écossaise ${ }^{16}$.

C'est paradoxalement chez les détracteurs de Boèce que l'on trouve l'exposé le plus détaillé de cette version de la protohistoire écossaise. William Maitland, ancien marchand élu membre de la Royal Society en 1733 et de l'Antiquarian Society deux ans plus tard, consacre en effet plusieurs pages de son History and Antiquities of Scotland à différents récits du peuplement de l'Écosse et notamment aux légendes de Partholamus et de Gadel dont il cite la version de l'historien irlandais Geoffrey Keating ${ }^{17}$ qu'il réfute avec virulence : «Of all the writings that went by

14. Voir Sir Th. Urquhart, Pantochronacanon (1652), réédité au XVIII siècle par D. Herd (éd.), Tracts of the Learned and celebrated Antiquarian Sir Thomas Urquhart, Édimbourg, Charles Herriott, 1774; G. Buchanan ne l'évoque même pas dans son History of Scotland, Londres, Crouch, 1690; W. Nicolson, The Scottish Historical Library, Londres, Childe, 1702; R. Sibbald, A Collection of Several Treatises in Folio Concerning Scotland as it was of Old, and also in later Times, Édimbourg, Hamilton \& Balfour, 1739; W. Duncan, The History of the Lives and Reigns of the Kings of Scotland, Dublin, Harding, 1722; D. Scott, The History of Scotland... from the year of the World 3619 to the year of Christ 1726, Westminster, J. Cluer, 1727; A. Gordon, Itinerarium Septentrionale, or a Fourney Thro' most of the Counties of Scotland... in two Parts, Londres, pour l'auteur, 1726; W. Maitland, The History and Antiquities of Scotland from the earliest Account of Time... in two Volumes, Londres, Millar, 1757.

15. J. Wallace, The History of the Lives and Reigns of the Kings of Scotland from Fergus the First King, Dublin, 1722, notamment l'introduction et les pages 59-60; J. Brome, Travels over England, Scotland and Wales, Londres, A. Roper, 1700, voir p. 181-182; Ch. Cordiner, Remarkable Ruins and Romantic Prospects of North Britain, 2 vols, Londres, Taylor, 1795, voir notamment «Introductory Remarks on the Obelisks of North Britain on which there are Egyptian Symbols», «Egyptian Hieroglyphics Observable on Caledonian Obelisks», «Primæval Hieroglyphics», t. I, n. p.; Th. Innes, A Critical Essay on the Ancient Inhabitants of the Northern Parts of Britain and Scotland... in two Volumes, Londres, Innys, 1729.

16. Voir C. Kidd, Subverting Scotland's Past, ouvr. cité, p. 70-99; C. Kidd, British Identities Before Nationalism, Cambridge, Cambridge University Press, 1999, p. 123-145; A. I. Maciness, Union and Empire: The Making of the United Kingdom in 1707, Cambridge, Cambridge University Press, 2007, p. 13-50.

17. Voir G. Keating, General History of Ireland, translated from the Irish with Amendements by D. O'Connor, Londres, B. Creake, 1723. 
the name of history, surely this is the most ridiculous and absurd that was ever devised ${ }^{18}$. » Keating s'attache uniquement à décrire les étapes du peuplement de l'Irlande et bouleverse largement la chronologie de Boèce puisque les Irlandais de Gadel partent conquérir l'Écosse des Pictes dont Boèce situe l'arrivée deux siècles après celle des Égyptiens de son Gathelus. Cette version plus récente souligne la rivalité qui, au XVIII siècle, oppose l'Irlande à l'Écosse, chaque région ayant à cœur de démontrer qu'elle est la plus anciennement peuplée et la plus proche de la terre biblique, mais également que l'une a colonisé l'autre ${ }^{19}$. Si William Maitland rejette vigoureusement les explications de Boèce comme de Keating, c'est parce qu'il affirme que l'Écosse a été peuplée par des Celtes venus du continent, qui lui ont apporté leur langue, le gaélique, et leur système politique. Ce n'est pas l'extravagance de la thèse égyptienne qui motive son rejet puisqu'il fait siennes les théories de Pezron sur l'origine fabuleuse des Gaulois : installé à Londres, Maitland entend montrer que la population écossaise des Lowlands est à l'origine celle-là même qui s'est installée en Angleterre, les Pictes étant relégués dans les contrées sauvages du nord de l'Écosse.

\section{L'influence de la franc-maçonnerie et des antiquaires}

Quelle que soit la version retenue, et quelles que soient les raisons idéologiques qui sous-tendent le discours historique, la théorie égyptienne des origines du peuple écossais n'est pas sans lien avec les préoccupations de la franc-maçonnerie écossaise qui, nourrie de l'hermétisme kabbalistique de la Renaissance, se développe véritablement à partir du $\mathrm{XVII}^{\mathrm{e}}$ siècle et connaît son âge d'or au siècle suivant, période à laquelle la question de l'origine égyptienne de la franc-maçonnerie est âprement discutée en Grande-Bretagne comme sur le continent. Bien avant la campagne d'Égypte de 1798 ou l'émergence, surtout en France, de rites maçonniques «égyptiens» comme le rite de Misraïm ou rite oriental de Memphis, les premières loges qui se constituent en Écosse au XVII siècle s'appuient sur une tradition hermétique qui fait du hiéroglyphe égyptien le véhicule symbolique des secrets de l'univers. Ce lien se double d'un mythe fondateur qui fait remonter l'origine de la franc-maçonnerie au déluge et qui fait d'Hermès celui qui, ayant préservé le savoir ancien des bâtisseurs, le transmet aux survivants. Cette histoire, commencée avec

18. W. Maitland, The History and Antiquities of Scotland, ouvr. cité, p. 10.

19. Voir au sujet de l'Irlande : Cl. O'Halloran, Golden Ages and Barbarous Nations, Antiquarian Debate and Cultural Politics in Ireland, c. 1750-1800, Cork, Cork University Press, 2004. 
le déluge, se poursuit en Égypte, où Abraham et son épouse sont venus enseigner les sept sciences. Confrontée à la surpopulation, une partie de la population égyptienne doit quitter le pays, non sans avoir reçu d'Euclide un enseignement complet portant sur la géométrie et l'art des bâtisseurs, censé leur permettre de s'établir ailleurs dans le monde ${ }^{20}$.

Il est aisé de rapprocher les deux mythes fondateurs et de voir dans le Gathelus des chroniques l'un des bâtisseurs exilés de la franc-maçonnerie. La ville égyptienne de Memphis, que celui-ci part défendre avec l'armée de Pharaon, est elle aussi étroitement liée à l'histoire de la francmaçonnerie. Pour Jacques-Étienne Marconis de Nègre, fondateur du Rite oriental de Memphis en 1839, l'apôtre Jacques aurait converti un prêtre séraphique d'Alexandrie nommé Ormus qui fonda ensuite en Égypte une société initiatique, «Les sages de la lumière». Des siècles plus tard, les descendants des Esséniens liés à Ormus et à ses disciples communiquèrent leurs connaissances aux chevaliers de Palestine et des maçons orientaux venus à Édimbourg en 1140 seraient à l'origine de la maçonnerie écossaise ${ }^{21}$.

Deux ouvrages largement diffusés en Europe ont par ailleurs renforcé le lien entre l'Égypte et la franc-maçonnerie et contribué à présenter l'Égypte comme le berceau de tous les savoirs : Séthos, le roman de l'abbé Terrasson publié en France en 1731 et traduit en anglais l'année suivante, mais surtout et bien avant lui l'EEdipus Egyptiacus du jésuite allemand Athanase Kircher, publié à Rome entre 1652 et $1655^{22}$. Le premier, ouvrage de fiction présenté comme la traduction d'un récit en grec ancien, présente une Égypte largement marquée par l'ésotérisme à travers l'initiation de Séthos aux mystères d'Isis. Très proche des traditions maçonniques, ce roman est réputé avoir inspiré à Emanuel Schikaneder certains éléments du livret de La Flûte enchantée.

L'ouvrage de Kircher, teinté de néo-platonisme, cherche à mettre en relief la dimension symbolique des hiéroglyphes, que l'érudit jésuite affirme être en mesure de déchiffrer. Malgré le mysticisme hermétique qui caractérise l'ouvrage de Kircher, Erik Iversen souligne qu'il fut le premier à avoir eu l'intuition de la valeur phonétique des hiéroglyphes,

20. Voir D. Stevenson, The Origins of Freemasonry: Scotland's Century 1590-1710, Cambridge, Cambridge University Press, 1988.

21. Voir J.-É. Marconis de Nègre, Le Sanctuaire de Memphis, ou Hermès, Paris, Bruyer, 1848, p. 5-6.

22. A. Kircher, Edipus Egyptiacus, Rome, Mascardi, 1652-1654; J. Terrasson, Séthos, histoire, ou Vie tirée des monuments anecdotes de l'ancienne Égypte, traduite d'un manuscrit grec, 3 vols, Paris, Guérin, 1731 ; pour l'édition anglaise: The Life of Sethos. Taken from private memoirs of the Ancient Egyptians, trad. Lediard, 2 vols, Londres, Walthoe, 1732. 
voyant dans l'écriture copte une survivance des hiéroglyphes anciens ${ }^{23}$. Il publie presque à la même époque une somme consacrée à la description et au déchiffrement des hiéroglyphes observés sur l'obélisque Pamphili à Rome, étude qui lui est commandée par le pape Innocent X et dans laquelle il applique ses propres théories.

$\mathrm{Au}$ début du XVIII ${ }^{\mathrm{e}}$ siècle, l'excavation de statues et d'inscriptions égyptiennes à Rome place l'Égypte au centre des préoccupations des antiquaires et de nombreuses collections sont constituées, en Angleterre comme en Écosse, tandis que les expéditions se succèdent en Égypte même. Des ouvrages illustrés portant sur la description de l'Égypte sont publiés en France et en Angleterre, comme L'Antiquité expliquée de Bernard de Montfaucon ou les descriptions de l'Égypte de Benoît de Maillet, Richard Pococke ou Frederik Norden ${ }^{24}$. Certains auteurs s'intéressent par ailleurs autant à l'histoire ancienne qu'à l'histoire écossaise, comme Alexander Gordon. Secrétaire de la Society of Antiquaries de Londres à partir de 1736 puis secrétaire de l'Egyptian Society dont William Stukeley et Richard Pococke étaient membres, l'Écossais Gordon est à la fois l'auteur d'une description de l'Écosse — Itinerarium Septentrionale — et de plusieurs ouvrages sur les antiquités égyptiennes et notamment les hiéroglyphes observables sur des momies détenues par des collectionneurs privés ${ }^{25}$.

\section{Relecture et réécriture : le cas de la pierre de Forres}

Un monument du passé écossais, la pierre de Forres ou Forres Pilar, décrite et interprétée dans plusieurs ouvrages au XVIII ${ }^{\mathrm{e}}$ siècle, illustre assez bien la façon dont cette tradition égyptienne a pu participer directement ou indirectement d'une réécriture et d'une relecture au sens propre du passé de l'Écosse. Hector Boèce est apparemment le premier à en parler sous le nom de Sueno's Stone. Il affirme qu'elle a été érigée pour commémorer

23. E. Iversen, The Myth of Egypt and its Hieroglyphs in European Tradition, Copenhague, 1961 ; Princeton, Princeton University Press, 1993 : voir notamment, p. 88-123.

24. B. de Montfaucon, L'Antiquité expliquée et représentée en figures, 10 vols, Paris, Delaulne, 1719 , traduit en anglais par D. Humphreys : Antiquity Explained, 5 vols, Londres, J. Tonson, 1721 ; B. de Maillet, Description de l'Égypte, Paris, Genneau et Rollin, 1735; R. Pococke, A Description of the East and some Other Countries, 3 vols, Londres, pour l'auteur, 1743; Fr. L. Norden, Voyage d'Égypte et de Nubie, Copenhague, imprimerie de la Maison royale des orphelins, 1755, traduit en anglais par P. Templebaum, Travels in Egypt and Nubia, 2 vols, Londres, L. Davis, 1757.

25. A. Gordon, Essay Towards Explaining the Hieroglyphical Figures on the Coffin of the Ancient Mummy Belonging to Captain W. Lethieullier, Londres, pour l'auteur, 1737; Twenty Five Plates of all the Egyptian Mummies, and other Egyptian Antiquities in England, Londres, pour l'auteur, 1739; pour Itinerarium Septentrionale, ouvr. cité, voir référence note 13. 
la victoire des Écossais contre les soldats du roi norvégien Sueno, empoisonnés nuitamment par Duncan et Macbeth. Alexander Gordon en donne sans doute la première description complète et la première représentation iconographique dans son Itinerarium Septentrionale. Il écrit à propos des dessins qu'il observe sur cette pierre :

Taking it for granted that the Scots never came from Ægypt, yet this hieroglyphical way of representing facts, is uncontrovertedly like the Ægyptian fashion, and was without doubt, invented to transmit their most memorable actions to posterity, in those dark ages, when literature, and erudition, were scarce known; and I am not altogether without hopes, that, as I have the honour of presenting these things first to the view of the world, my work may be more candidly receiv'd [...]; and who also knows but some future critick of antiquity, from their very symbols, and hieroglyphicks, here exhibited, may discover still stronger circumstances, to convince the enemies of the Scots, that their nation is not quite destitute of all vouchers of historical facts ${ }^{26}$.

Il ne s'agit pas pour lui de chercher dans l'obélisque de Forres (c'est ainsi qu'il est nommé sur une gravure plus tardive de William Daniell ${ }^{27}$ ) la preuve de l'origine égyptienne du peuple écossais, mais d'affirmer que le système symbolique gravé sur cette pierre présente le même degré de sophistication que les hiéroglyphes sacrés d'Égypte et que la volonté manifeste de transmettre un savoir et un patrimoine à l'épreuve du temps est l'indice d'un haut degré de civilisation (voir fig. 1). Gordon n'a d'ailleurs aucun doute sur l'origine nordique de cette pierre et raille les théories de Boèce : «I must believe them to be remains of the later incursions of the Danes, and other Northern Nations. Hector Boetius, in one of his particular fancies, thinks them relicks of the Egyptian fashion ${ }^{28}$.»

Des années plus tard, le géologue français Barthélémy Faujas de SaintFond rapproche lui aussi les motifs sculptés qu'il observe de ceux relevés sur les tombeaux d'Égypte sans pour autant en tirer de conclusion particulière : «Ces sculptures, qui paraissent être des tombeaux, ont un style singulier qui a des rapports avec celui des Égyptiens; ce sont des figures emmaillotées, à l'instar de celles des boîtes des momies ${ }^{29}$.» S'il n'est plus question de relier les premiers Écossais au peuple d'Égypte, l'utilisation d'un vocabulaire employé pour décrire les antiquités égyptiennes ainsi

26. A. Gordon, Itinerarium Septentrionale, ouvr. cité, p. 160.

27. W. Daniell et R. Ayton, Voyage Round Great Britain, 8 vols, Londres, Longman, 1814-1825, vol. 5, p. 82.

28. A. Gordon, Itinerarium Septentrionale, ouvr. cité, p. 159.

29. B. Faujas de Saint-Fond, Voyage en Angleterre, en Écosse et aux îles Hébrides, 2 vols, Paris, H. J. Jansen, 1797, t. II, p. 226. 


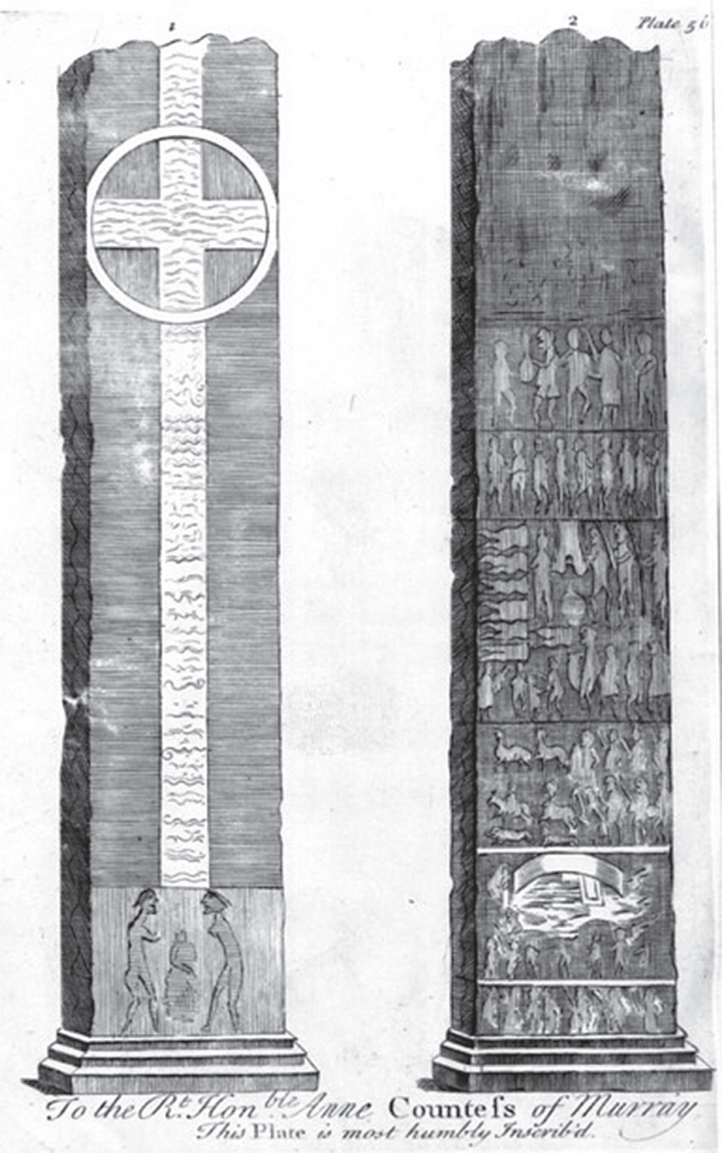

Fig. 1. - Gordon, «Forres Pillar».

que les comparaisons constantes entre les pierres levées écossaises et les obélisques égyptiens entretiennent, cependant, une certaine confusion, d'autant plus que le relevé des motifs gravés sur la pierre de Forres effectué par Gordon fait apparaître des créatures fabuleuses, proches du centaure, face auxquelles l'auteur lui-même avoue sa perplexité :

The whole, above ground, is divided into seven compartments [...] The second contains sundry figures, but most of them defac'd. In the third I discovered several of a monstrous form, resembling four-footed beasts, with humane heads and others, of Men standing by them. In the fourth division, 
are six or seven ensigns, or standards, with some figures, holding obscure weapons in their hands ${ }^{30}$.

En 1769, le naturaliste Thomas Pennant décrit à son tour sommairement la pierre de Forres : «Near Forres, on the road-side, is a vast column [...] On one side are numbers of rude figures of animals and armed men, with colours flying: some of the men seemed bound like captives ${ }^{31}$.» Quelques décennies plus tard, Charles Cordiner s'intéresse lui aussi la fameuse pierre et reproche à Gordon l'interprétation floue qu'il en donne et son manque de fidélité à l'original. Ministre épiscopalien et antiquaire, Cordiner est l'auteur de deux ouvrages importants : Antiquities and Scenery of the North of Scotland et Remarkable Ruins and Romantic Prospects of North Britain $^{32}$. Il consacre une lettre à l'obélisque de Forres dans le premier livre et pas moins de trois articles dans le second, associant des gravures à chacune de ses descriptions. À plus d'un demi-siècle de distance, il accuse Gordon d'avoir représenté des motifs qui ne se trouvent pas sur la pierre et plus précisément d'avoir représenté des créatures fantastiques et orientalisantes qui paradoxalement n'apparaissent pas toutes sur la planche de Gordon : «For he has given a sow, a sphinx, and a centaur, and some camels with human heads - instead of fixed men on horseback, who are flying before a pursuing enemy on foot ${ }^{33}$.» Gordon avait certes cru voir des centaures, mais c'est Cordiner qui voit sur la gravure de Gordon un bestiaire fabuleux qu'il est aisé pour le lecteur de rapprocher du désert égyptien. S'il semble dénigrer Gordon, il cite pourtant largement son texte, reprenant également les remarques de Nicholson citées par ce dernier dans un chapitre de Remarkable Ruins intitulé «Introductory Remarks on the Obelisks of North Britain, on Which are Egyptian Symbols », Cordiner entend surtout se rattacher à une tradition historiographique prestigieuse — «our most intelligent historians» - pour souligner sa propre légitimité et mettre en avant sa lecture personnelle de l'obélisque de Forres, dont il donne une illustration précise dans chacun de ses deux ouvrages. Contempteur de la théorie égyptienne du peuplement de l'Écosse propagée par les chroniqueurs médiévaux, Cordiner n'en reste pas moins convaincu que les «hiéroglyphes» trouvés sur les «obélisques» écossais s'inscrivent dans une tradition symbolique directement héritée de l'Égypte et insiste sur l'étrange affinité («strange affinity») et l'inexplicable ressemblance («unaccountable resemblance») qui existe entre

30. A. Gordon, Itinerarium Septentrionale, ouvr. cité, p. 158.

31. Th. Pennant, A Tour of Scotland, 1769, Chester, John Monk, 1771, p. 130-131.

32. Ch. Cordiner, Antiquities and Scenery of the North of Scotland, in a Series of Letters to Thomas Pennant, Londres, s. n., 1780; Remarkable Ruins, voir note 14.

33. Ch. Cordiner, «Forres Pillar», dans Remarkable Ruins, ouvr. cité, vol. 1, n. p. 
les monuments écossais et égyptiens. Il annonce ainsi ses intentions, sans omettre d'évoquer la dimension initiatique de son travail de déchiffrement :

It was not until after the maturest deliberation, and frequent canvassing of the subjects with men of knowledge and learning, that it was resolved on to attempt the description of those Caledonian Monuments, which involve mysterious allusions in their sculpture: because, in order to develop the hidden meaning of these abstruse symbols, it is requisite to go deep into the labyrinth of hieroglyphical records, the intricate windings of which, perhaps, 'tis only those of singular taste and penetration, and who have much leisure time at command, that have either ambition or perseverance to unravel ${ }^{34}$.

C'est peut-être en tant que franc-maçon, ou au moins en tant que familier du symbolisme hermétique, que Cordiner se penche sur la pierre levée de Forres et sur d'autres reliques du passé écossais. Marie-Cécile Révauger a montré que de nombreux membres de la Society of Antiquarians étaient francs-maçons et en France, à la fin du XVIII ${ }^{\mathrm{e}}$ siècle et au début du XIX ${ }^{\mathrm{e}}$, certains auteurs ont également cherché à rattacher certaines pierres gravées aux bas-reliefs égyptiens, comme Alexandre Lenoir, érudit, muséographe et franc-maçon, qui écrit à propos des portiques des cathédrales gothiques : «La plupart des figures emblématiques qui décorent les portails de nos anciennes églises sont du même genre; nous les considérons comme des espèces d'hyérogliphes [sic] semblables à ceux des Égytiens $[\text { sic }]^{35}$.» Les symboles maçonniques abondent, en effet, dans Remarkable Ruins dont la page de garde est ornée d'un médaillon gravé par Peter Mazell et surchargé de symbolique maçonne : le delta lumineux, les colonnes du temple, les trois incarnations du feu sacré ou encore la croix de Saint-André barrée qui est à la fois celle de l'Écosse mais aussi un symbole de vie et de perfection dans la tradition maçonnique.

S'arrêtant sur un autre groupe de pierres gravées trouvées dans la campagne écossaise à Aberlemny (voir fig. 2), Cordiner, enthousiaste, livre au lecteur son interprétation à partir d'une citation de Kircher :

"The circles, then, included within the Triune line, finished at either end with a sceptral emblem, or ensigne of EFFICIENT POWER, was the expressive symbol of the DIVINE DOMINION extended to heaven and earth, and the whole

34. Ch. Cordiner, «Introductory Remarks», dans Remarkable Ruins, ouvr. cité, n. p.

35. Voir M.-G. Révauger, Le Fait maçonnique au XVIII e siècle en Grande-Bretagne et aux États-Unis, Paris, EDIMAF, 1990. A. Lenoir, Musée et monuments français, 2 vols, Paris, Guillemet, 1802, t. II, p. 37. Voir également: A. Lenoir, Nouvelle explication des hiéroglyphes ou des anciennes allégories sacrées des Égyptiens, utile à l'intelligence des monuments mythologiques des autres peuples, 4 vols, Paris, Musée des monuments français, 1809-1821. En Angleterre, l'évêque Warburton effectue le même rapprochement. Voir à ce sujet : J. Baltrusaitis, La Quête d'Isis (1985), Paris, Flammarion, 1990. 


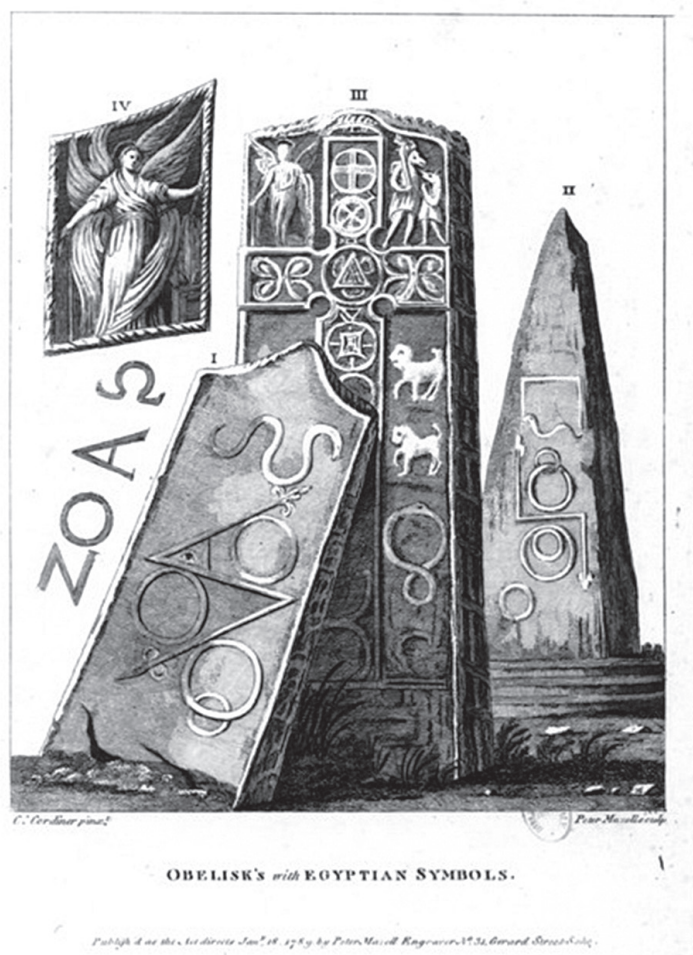

Fig. 2. - Cordiner, «Obelisks with Egyptian symbols».

circle of the year." While the sacred EYE, within the triangle, gives a thought of sublimity, which is impossible to heighten.

We have here then, on a CALEDONIAN monument, one of the most original and sacred symbols of the EASTERN WORLD; in the mystic lines of which, those that are thoroughly versed in antiquity can be at no loss to discern the rudiments of the Alpha and Omega, and other letters of the primæval alphabet, as in the extracted NOA $\Omega^{36}$ !

Si les Égyptiens n’ont pas colonisé l'Écosse, et si la parenté entre les monuments écossais et égyptiens n'est pas purement accidentelle, l'explication doit se trouver ailleurs que dans les fables médiévales. Celle qui est avancée par Cordiner mêle la science initiatique des Égyptiens et la vérité

36. Ch. Cordiner, «Ægyptian Hieroglyphics Observable on Caledonian Obelisks», dans Remarkable Ruins, n. p. La citation de Kircher est tirée de Obeliscus Pamphilius, Rome, Grignani, 1650, p. 158. 
universelle du message colporté par les premiers missionnaires chrétiens, et s'il tourne le dos aux chroniqueurs du Moyen Âge et de la Renaissance c'est pour promouvoir l'idée d'une parenté spirituelle et non plus d'un simple lignage :

The evidence of the sublime allusions, here offered to view, is not therefore derived from the legendary tales of early historians; nor from the dubious theories founded in fiction and favoured by credulity; but from the authentic testimony of sculptured granite and marble, and the venerable importance of primæval symbols.

It is therefore a pleasing reflection, that so many of these obeLISKs held sacred by their antiquity, and the traditionary value of their contents, have so long remained the boast and glory of the CALEDONIAN plains ${ }^{37}$.

Quelle que soit la méthode ou le discours historiographique, l'ambition de Boèce est finalement sensiblement la même que celle de l'antiquaire de la fin du XVIII ${ }^{\mathrm{e}}$ siècle : il s'agit d'affirmer que l'Écosse est, comme l'Égypte ancienne, davantage qu'un peuple, une civilisation qui a su développer pendant sa longue histoire ce «genius of language and of arts» cher à James Dunbar. C'est une Écosse initiée, proche des mystères et du sacré qui transparaît dans les réécritures de son passé antique : une mythologie spirituelle qui contraste volontairement avec la sauvagerie que les chroniqueurs romains ont attribuée à un peuple invaincu.

Une partie des historiens écossais des Lumières n'accordaient certainement plus crédit au mythe égyptien du peuplement de l'Écosse, mais la manière dont nombre d'entre eux insistent sur les points communs entre les restes du passé écossais, que l'on redécouvre alors, et les monuments égyptiens, parlant indistinctement dans un cas comme dans l'autre d'obélisques et de hiéroglyphes, souligne surtout la proximité qui existe entre les préoccupations des chroniqueurs de la Renaissance et celles des historiens du XVIII ${ }^{\mathrm{e}}$ siècle. Les uns comme les autres entendent conjurer la marginalité de l'Écosse et cherchent moins à la rapprocher d'une Égypte fantasmatique et lointaine que d'une Europe savante dont ils partagent les interrogations culturelles et spirituelles.

37. Ch. Cordiner, «Introductory Remarks», dans Remarkable Ruins, ouvr. cité, n. p. 


\section{Références bibliographiques}

\section{Sources primaires}

Bò̀ce Hector, Scotorum Historice a prima gentis origine, Paris, 1526 ; traduction anglaise en 2 vols de raphael Hollinshed, The Description of Scotland Written by H. Beothius in Latin... Translated into the Scotish [sic] Speech by John Belledon... and now finally into English by R. H., Londres, Harrison, 1577.

Brome James, Travels over England, Scotland and Wales, Londres, A. Roper, 1700.

Buchanan George, History of Scotland, Londres, Crouch, 1690.

Cassan Jacques de, Les Dynasties, ou Traité des anciens rois des Gaulois et des François depuis le Déluge successivement jusqu'au roi Mérovée, Paris, s. d.

Cordiner Charles, Remarkable Ruins and Romantic Prospects of North Britain, 2 vols, Londres, Taylor, 1795.

-, Antiquities and Scenery of the North of Scotland, in a Series of Letters to Thomas Pennant, Londres, s. n., 1780.

Daniell William et Ayton Richard, Voyage Round Great Britain, 8 vols, Londres, Longman, 1814-1825.

Duncan William, The History of the Lives and Reigns of the Kings of Scotland, Dublin, Harding, 1722.

Faujas de Saint-Fond Barthélémy, Voyage en Angleterre, en Écosse et aux îles Hébrides, 2 vols, Paris, H. J. Jansen, 1797.

FORDUn John of, Chronica Gentis Scotorum (Scotichronicon). La première édition imprimée paraît dans le troisième volume des Scriptores Quindecim de Thomas Gale, 1691.

Gordon Alexander, Essay Towards Explaining the Hieroglyphical Figures on the Coffin of the Ancient Mummy Belonging to Captain W. Lethieullier, Londres, pour l'auteur, 1737.

-, Itinerarium Septentrionale, or a Fourney Thro' most of the Counties of Scotland... in two Parts, Londres, pour l'auteur, 1726.

- Twenty Five Plates of all the Egyptian Mummies, and other Egyptian Antiquities in England, Londres, pour l'auteur, 1739.

Gray Sir Thomas, Scalacronica (1355), Édimbourg, pour le Maitland Club, 1836.

Innes Thomas, A Critical Essay on the Ancient Inhabitants of the Northern Parts of Britain and Scotland... in two Volumes, Londres, Innys, 1729.

Keating Geoffrey, General History of Ireland, translated from the Irish with Amendements by D. O'Connor, Londres, B. Creake, 1723.

Kircher Athanase, Obeliscus Pamphilius, Rome, Grignani, 1650.

—, Edipus Agyptiacus, Rome, Mascardi, 1652-1654. 
Lemaire de Belges Jean, Les Illustrations de Gaule et singularités de Troye, Paris, 1512.

LenoIr Alexandre, Musée et monuments français, 2 vols, Paris, Guillemet, 1802.

_- Nouvelle explication des hiéroglyphes ou des anciennes allégories sacrées des Égyptiens, utile à l'intelligence des monuments mythologiques des autres peuples, 4 vols, Paris, Musée des monuments français, 1809-1821.

MacPherson James, Fragments of Ancient Poetry, Collected in the Highlands of Scotland, and translated from the Galic or Erse Language, Édimbourg, Hamilton \& Balfour, 1760.

Maillet Benoît de, Description de l'Égypte, Paris, Genneau et Rollin, 1735.

Maitland William, The History and Antiquities of Scotland from the earliest Account of Time... in two Volumes, Londres, Millar, 1757.

Marconis de NèGre Jacques-Étienne, Le Sanctuaire de Memphis, ou Hermès, Paris, Bruyer, 1848.

Montfaucon Bernard de, L'Antiquité expliquée et représentée en figures, 10 vols, Paris, Delaulne, 1719; traduit en anglais par David Humphreys, Antiquity Explained, 5 vols, Londres, J. Tonson, 1721.

Nicolson William, The Scottish Historical Library, Londres, Childe, 1702.

Norden Frederik Ludvig, Voyage d'Égypte et de Nubie, Copenhague, imprimerie de la Maison royale des orphelins, 1755; traduit en anglais par Peter Templebaum, Travels in Egypt and Nubia, 2 vols, Londres, L. Davis, 1757.

Pennant Thomas, A Tour of Scotland, 1769, Chester, John Monk, 1771.

Pezron Paul-Yves, Antiquité de la nation et de la langue des Celtes, autrement appelez Gaulois, Paris, Jean Boudot, 1703; pour la version anglaise : The Antiquities of Nations; more particularly of the Celte or Gauls, taken to be originally the same people as our ancient Britains, Londres, S. Ballard, 1706.

Pococke Richard, A Description of the East and some Other Countries, 3 vols, Londres, pour l'auteur, 1743.

ScotT David, The History of Scotland... from the year of the World 3619 to the year of Christ 1726, Westminster, J. Cluer, 1727.

Sibbald Robert, A Collection of Several Treatises in Folio Concerning Scotland as it was of Old, and also in later Times, Édimbourg, Hamilton \& Balfour, 1739.

Terrasson Jean, Séthos, histoire, ou Vie tirée des monuments anecdotes de l'ancienne Égypte, traduite d'un manuscrit grec, 3 vols, Paris, Guérin, 1731 ; pour l'édition anglaise: The Life of Sethos. Taken from private memoirs of the Ancient Egyptians, trad. Lediard, 2 vols, Londres, Walthoe, 1732.

URQuHart Sir Thomas, Pantochronacanon (1652), réédité au XVIII siècle par D. Herd (éd.), Tracts of the Learned and celebrated Antiquarian Sir Thomas Urquhart, Édimbourg, Charles Herriott, 1774. 
Wallace James, An Account of the Islands of Orkney, to which is added an Essay on the Thule of the Ancients, Londres, J. Tonson, 1700.

- The History of the Lives and Reigns of the Kings of Scotland from Fergus the First King, Dublin, 1722.

\section{Sources secondaires}

Baltrusaitis Jurgis, La Quête d'Isis (1985), Paris, Flammarion, 1990.

Iversen Erik, The Myth of Egypt and its Hieroglyphs in European Tradition, Copenhague, 1961 ; Princeton, Princeton University Press, 1993.

KIDD Colin, Subverting Scotland's Past: Scottish Whig Historians and the Creation of an Anglo-British Identity, 1689-c. 1830, Cambridge, Cambridge University Press, 1993.

- British Identities Before Nationalism: Ethnicity and Nationhood in the Atlantic World, 1600-1800, Cambridge, Cambridge University Press, 1999.

Macinnes Allan I., Union and Empire: The Making of the United Kingdom in 1707, Cambridge, Cambridge University Press, 2007.

Matthews William, «The Egyptians in Scotland: The Political History of a Myth», Viator, 1, 1970, p. 289-306.

O'Halloran Clare, Golden Ages and Barbarous Nations, Antiquarian Debate and Cultural Politics in Ireland, c. 1750-1800, Cork, Cork University Press, 2004.

Révauger Marie-Cécile, Le Fait maçonnique au XVIII siècle en Grande-Bretagne et aux États-Unis, Paris, EDIMAF, 1990.

Stevenson David, The Origins of Freemasonry: Scotland's Century 1590-1710, Cambridge, Cambridge University Press, 1988.

Talon Alain (éd.), Le Sentiment national dans l'Europe méridionale aux XVI et XVII ${ }^{e}$ siècles, Madrid, Casa Velasquez, 2007. 